\title{
Supersymmetric Calogero models by gauging
}

\author{
Sergey Fedoruk, ${ }^{1}$ Evgeny Ivanov, ${ }^{1}$ and Olaf Lechtenfeld ${ }^{2}$ \\ ${ }^{1}$ Bogoliubov Laboratory of Theoretical Physics, JINR, 141980 Dubna, Moscow Region, Russia \\ ${ }^{2}$ Institut für Theoretische Physik, Leibniz Universität Hannover, Appelstraße 2, D-30167 Hannover, Germany
}

(Received 23 January 2009; published 18 May 2009)

\begin{abstract}
New superconformal extensions of $d=1$ Calogero-type systems are obtained by gauging the $\mathrm{U}(n)$ isometry of matrix superfield models. We consider the cases of $\mathcal{N}=1, \mathcal{N}=2$, and $\mathcal{N}=4$ as onedimensional supersymmetries. The bosonic core of the $\mathcal{N}=1$ and $\mathcal{N}=2$ models is the standard conformal $A_{n-1}$ Calogero system, whereas the $\mathcal{N}=4$ model is an extension of the U(2)-spin Calogero system.
\end{abstract}

DOI: 10.1103/PhysRevD.79.105015

PACS numbers: 12.60.Jv, 02.10.Yn, 02.30.Ik, 11.30.Pb

\section{INTRODUCTION}

Superconformal extensions of the Calogero model [1] provide nice examples of integrable supersymmetric quantum-mechanical systems and as such are of vast interest from various points of view (see [2,3] for the survey of physical applications of the Calogero model). In particular, by a conjecture of Gibbons and Townsend [4], $\mathcal{N}=4$ superconformal models might be closely related to the $\mathrm{M}$ theory. While the $\mathcal{N}=2$ super-Calogero models for any number of interacting particles were constructed in full generality rather long ago [5] (see also [6,7]), until now there is not such an exhaustive understanding of the generic $\mathcal{N}=4$ models despite the existence of extensive literature on this subject (see e.g. [6-11]). It seems important to develop some universal approach to superconformal Calogero-type models including the $\mathcal{N}=4$ ones.

The purpose of this paper is to present a candidate approach of this type suitable for an arbitrary number of interacting particles. It is based on the superfield gauging of some non-Abelian isometries of the $d=1$ field theories. This gauging procedure was worked out in [12] to understand off-shell dualities between $d=1$ supermultiplets with different sets of physical bosonic components.

Our starting point is the nice interpretation of the bosonic $n$-particle Calogero model as a $\mathrm{U}(n), d=1$ gauge theory [13] (see also [14-16]). In the formulation of [13], the model is described by the Hermitian $n \times n$-matrix field $X_{a}^{b}(t),\left(\bar{X}_{a}^{b}\right)=X_{b}^{a}$, the complex $n$-plet $Z_{a}(t), \bar{Z}^{a}=\left(\bar{Z}_{a}\right), a$, $b=1, \ldots, n$, and $n^{2}$ nonpropagating "gauge fields" $A_{a}^{b}(t)$, $\left(\bar{A}_{a}^{b}\right)=A_{b}^{a}$. The action reads

$$
S_{0}=\int d t\left[\operatorname{Tr}(\nabla X \nabla X)+\frac{i}{2}(\bar{Z} \nabla Z-\nabla \bar{Z} Z)+c \operatorname{Tr} A\right]
$$

where the covariant derivatives are defined as

$$
\nabla X=\dot{X}+i[A, X], \quad \nabla Z=\dot{Z}+i A Z .
$$

The real constant $c$ of the Calogero interaction comes out from a Fayet-Iliopoulos (FI) term in (1).
The action (1) is invariant with respect to the local $\mathrm{U}(n)$ transformations, $g(\tau) \in \mathrm{U}(n)$,

$$
X \rightarrow g X g^{\dagger}, \quad Z \rightarrow g Z, \quad A \rightarrow g A g^{\dagger}+i \dot{g} g^{\dagger},
$$

and we can fully fix the $\mathrm{U}(n)$ gauge freedom by choosing

$$
X_{a}^{b}=x_{a} \delta_{a}^{b}, \quad \bar{Z}^{a}=Z_{a} .
$$

Inserting these gauge conditions and the algebraic equations of motion $\left(Z_{a}\right)^{2}=c$ (which implies $c>0$ ) and $A_{a}^{b}=$ $Z_{a} Z_{b} /\left[2\left(x_{a}-x_{b}\right)^{2}\right], a \neq b$, back into the action (1), we arrive at the standard Calogero action

$$
S_{0}=\int d t\left[\sum_{a} \dot{x}_{a} \dot{x}_{a}-\sum_{a \neq b} \frac{c^{2}}{4\left(x_{a}-x_{b}\right)^{2}}\right]
$$

as a fixed gauge of (1). Note the important role of the auxiliary $\mathrm{U}(n)$ multiplet $Z$ with the $d=1$ Wess-Zumino (WZ) action in (1) for recovering the Calogero action.

The original action (1) is invariant under the $d=1$ conformal $\mathrm{SO}(1,2)$ transformations: $\delta t=a, \delta X_{a}^{b}=$ $\frac{1}{2} \dot{a} X_{a}^{b}, \delta Z_{a}=0, \delta A_{a}^{b}=-\dot{a} A_{a}^{b}$, where $a(t)$ obeys the constraint $\dddot{a}=0$. This property implies the well-known conformal invariance of the eventual Calogero model.

Our approach is a minimal superfield generalization of this bosonic $\mathrm{U}(n)$ gauging. Requiring the supersymmetric gauge models to possess $\mathcal{N}$-extended superconformal symmetry essentially constrains the structure of the corresponding actions and allows one to reveal, in their bosonic sector, either the standard Calogero model (4) (in the cases of $\mathcal{N}=1$ and $\mathcal{N}=2$ ) or the $\mathrm{U}(2)$-spin Calogero model $[3,17,18]$ modified by a conformal potential for the centerof-mass coordinate (in the $\mathcal{N}=4$ case). In this short paper we outline the basic features of our construction, leaving details, quantization, and comparison with the previously known superextended Calogero models for a longer paper.

\section{II. $\mathcal{N}=1$ SUPERSYMMETRIC EXTENSION}

We use the Grassmann-even Hermitian $\mathcal{N}=1$ matrix superfield $\mathcal{X}_{a}^{b}(t, \theta),(\mathcal{X})^{\dagger}=\mathcal{X}$, belonging to the adjoint representation of $\mathrm{U}(n)$, as well as the Grassmann-even 
complex $\mathcal{N}=1$ superfield $Z_{a}(t, \theta), \bar{Z}^{a}(t, \theta)=\left(Z_{a}\right)^{\dagger}$, in the fundamental of $\mathrm{U}(n)$. The spinor and time derivatives,

$$
D=\partial_{\theta}+i \theta \partial_{t}, \quad\{D, D\}=2 i \partial_{t}
$$

are gauge covariantized by the anti-Hermitian Grassmannodd connections $\mathcal{A}_{a}^{b}(t, \theta),(\mathcal{A})^{\dagger}=-\mathcal{A}$ :

$$
\begin{gathered}
\mathcal{D} \mathcal{X}=D \mathcal{X}+i[\mathcal{A}, \chi], \quad \nabla_{t} \mathcal{X}=-i \mathcal{D} \mathcal{D} \mathcal{X}, \\
\mathcal{D} \mathcal{Z}=D \mathcal{Z}+i \mathcal{A} \mathcal{Z} .
\end{gathered}
$$

The minimal gauge invariant action has the following form $\left(\mu_{1}=d t d \theta\right)$ :

$$
\begin{aligned}
S_{1}= & -i \int \mu_{1}\left[\operatorname{Tr}\left(\nabla_{t} \mathcal{X} \mathcal{D} X+c \mathcal{A}\right)\right. \\
& \left.+\frac{i}{2}(\bar{Z} \mathcal{D} Z-\mathcal{D} \bar{Z} Z)\right] .
\end{aligned}
$$

It is invariant under the local $\mathrm{U}(n)$ transformations:

$$
\begin{gathered}
\mathcal{X}^{\prime}=e^{i \tau} \chi e^{-i \tau}, \quad Z^{\prime}=e^{i \tau} \mathcal{Z}, \\
\mathcal{A}^{\prime}=e^{i \tau} \mathcal{A} e^{-i \tau}-i e^{i \tau} D e^{-i \tau},
\end{gathered}
$$

where $\tau_{a}^{b}(t, \theta) \in u(n)$ is the Hermitian matrix parameter. It is also invariant under the $\mathcal{N}=1$ superconformal group. Conformal supersymmetry $\delta^{\prime} t=-i \eta \theta t, \delta^{\prime} \theta=\eta t$ has the following realization on the involved superfields:

$$
\delta^{\prime} \mathcal{X}=-i \eta \theta \mathcal{X}, \quad \delta^{\prime} \mathcal{A}=i \eta \theta \mathcal{A}, \quad \delta^{\prime} \mathcal{Z}=0 .
$$

Its closure with the Poincare $\mathcal{N}=1, d=1$ supersymmetry yields the full $\mathcal{N}=1$ superconformal symmetry.

Because of the $\mathrm{U}(n)$ gauge invariance, we can choose the WZ gauge for the spinor connection:

$$
\mathcal{A}=i \theta A(t) .
$$

Substituting this into the action (5), integrating there over $\theta$ and eliminating the auxiliary fields by their equations of motion, we obtain

$$
S_{1}=S_{0}+S_{1}^{\Psi}, \quad S_{1}^{\Psi}=-i \operatorname{Tr} \int d t \Psi \nabla \Psi,
$$

where $\Psi=-i D \chi \mid$ is the matrix Grassmann-odd field and $\nabla \Psi=\dot{\Psi}+i[A, \Psi]$. The bosonic limit of (7) [and hence of (5)] is just the Calogero action (1). Its gauge $\mathrm{U}(n)$ symmetry is the residual symmetry of the WZ gauge (6).

An alternative supersymmetric gauge choice is

$$
\chi_{a}^{b}=0, a \neq b \Leftrightarrow \chi_{a}^{b}=\chi_{a} \delta_{a}^{b} ; \quad Z_{a}=\bar{Z}^{a} .
$$

In this gauge the model is described by $n^{2}$ real $\mathcal{N}=1$ superfields $\mathcal{A}_{a}^{b}, a \neq b$, and $\mathcal{X}_{a}$ (the superfields $Z_{a}$ and those on the diagonal of $\mathcal{A}_{a}^{b}$ are auxiliary). In the twoparticle case $(n=2)$, the resulting $\mathcal{N}=1$ system possesses an additional hidden $\mathcal{N}=1$ supersymmetry, so that the $n=2$ model is in fact $\mathcal{N}=2$ superconformal mechanics plus an $\mathcal{N}=2$ free multiplet corresponding to the center-of-mass motion. Starting with $n=3$, one gets new $\mathcal{N}=1$ superextensions of the $n$-particle Calogero models which cannot be recovered by any truncations of the standard $\mathcal{N}=2$ superextensions [5].

\section{III. $\mathcal{N}=2$ SUPERSYMMETRIC EXTENSION}

The relevant superfield content consists of the Hermitian matrix superfield $\mathcal{X}_{a}^{b}(t, \theta, \bar{\theta}),(\mathcal{X})^{\dagger}=\mathcal{X}$ with the off-shell field content $(\mathbf{1}, \mathbf{2}, \mathbf{1})$, and bosonic chiral $\mathrm{U}(n)$-fundamental superfield $Z_{a}\left(t_{L}, \theta\right), \bar{Z}^{a}\left(t_{R}, \bar{\theta}\right)=\left(Z_{a}\right)^{\dagger}, t_{L, R}=t \pm i \theta \bar{\theta}$,

$$
\bar{D} Z_{a}=0, \quad D \bar{Z}^{a}=0,
$$

with the field contents $(\mathbf{2}, \mathbf{2}, \mathbf{0})$. Here

$$
D=\partial_{\theta}+i \bar{\theta} \partial_{t}, \quad \bar{D}=-\partial_{\bar{\theta}}-i \theta \partial_{t}, \quad\{D, \bar{D}\}=-2 i \partial_{t} .
$$

The gauge prepotential is an $n \times n$ Hermitian matrix $V_{a}^{b}(t, \theta, \bar{\theta}),(V)^{\dagger}=V$. The action reads $\left(\mu_{2}=d t d^{2} \theta\right)$

$$
S_{2}=\int \mu_{2}\left[\operatorname{Tr}\left(\overline{\mathcal{D}} \chi e^{2 V} \mathcal{D} \chi e^{2 V}\right)+\frac{1}{2} \bar{z} e^{2 V} \mathcal{Z}-c \operatorname{Tr} V\right] \text {, }
$$

the gauge-covariant derivatives being defined as

$$
\begin{aligned}
& \mathcal{D} \mathcal{X}=D \mathcal{X}+e^{-2 V}\left(D e^{2 V}\right) \chi, \\
& \bar{D} \mathcal{X}=\bar{D} \mathcal{X}-\chi e^{2 V}\left(\bar{D} e^{-2 V}\right) .
\end{aligned}
$$

The action (10) is invariant under the local $\mathrm{U}(n)$ transformations:

$$
\chi^{\prime}=e^{i \lambda} \chi e^{-i \bar{\lambda}}, \quad Z^{\prime}=e^{i \lambda} Z, \quad e^{2 V^{\prime}}=e^{i \bar{\lambda}} e^{2 V} e^{-i \lambda},
$$

where $n^{2}$ complex gauge parameters $\lambda=\left(\lambda_{a}^{b}\right)$ are (anti) chiral superfields: $\lambda\left(t_{L}, \theta\right) \in u(n), \quad \bar{\lambda}\left(t_{R}, \theta\right)=(\lambda)^{\dagger} \in$ $u(n)$. The action is also invariant under the superconformal group $\mathrm{SU}(1,1 \mid 1)$. The conformal supersymmetry acts on the coordinates as

$$
\delta^{\prime} t=-i(\eta \bar{\theta}+\bar{\eta} \theta) t, \quad \delta^{\prime} \theta=\eta(t+i \theta \bar{\theta})
$$

and on the superfields as

$$
\delta^{\prime} \mathcal{X}=-i(\eta \bar{\theta}+\bar{\eta} \theta) \chi, \quad \delta^{\prime} V=0, \quad \delta^{\prime} Z=0 .
$$

The chirality conditions (9) are preserved by these transformations.

In the WZ gauge

$$
V(t, \theta, \bar{\theta})=-\theta \bar{\theta} A(t)
$$

the action (10) takes the form

$$
S_{2}=S_{0}+S_{2}^{\Psi}, \quad S_{2}^{\Psi}=-i \operatorname{Tr} \int d t(\bar{\Psi} \nabla \Psi-\nabla \bar{\Psi} \Psi),
$$

where $\Psi=D \mathcal{X} \mid$ is a Grassmann-odd field and

$$
\nabla \Psi=\dot{\Psi}+i[A, \Psi], \quad \nabla \bar{\Psi}=\dot{\bar{\Psi}}+i[A, \bar{\Psi}] .
$$


We see that the bosonic core of the action (13) exactly coincides with the Calogero action (1).

The action (13) is invariant with respect to the residual local bosonic $\mathrm{U}(n)$ transformations, defined by (2) and $\Psi \rightarrow g \Psi g^{\dagger}$; therefore we can choose the gauge (3). As a result we obtain an $\mathcal{N}=2$ superextension of the $n$-particle Calogero model. In the two-particle case $(n=$ 2) we found that the $\mathcal{N}=2$ supersymmetric gauged system actually describes $\mathcal{N}=4$ superconformal mechanics plus one $\mathcal{N}=4$ free multiplet corresponding to the center-of-mass motion, so that there is a hidden extra $\mathcal{N}=2$ symmetry in this case. For $n>2$ we obtain some new $\mathcal{N}=2$ extensions of the $n$-particle Calogero models with $n$ bosonic variables and $n \times n$ fermionic ones, as opposed to the standard $\mathcal{N}=2$ super-Calogero with $n$ complex fermions [5].

The presence of the matrix Grassmann-odd field $\Psi$ in the action (13) [and also in (7)] is imperative for $d=1$ supersymmetry and superconformal symmetry. Similar structures with the bosonic analogs of $\Psi$ appeared e.g. in $[15,16]$ in connection with the quantum Hall effect.

\section{IV. $\mathcal{N}=4$ SUPERSYMMETRIC EXTENSION}

This case surprisingly yields $\mathrm{U}(2)$-spin Calogero system $[17,18]$ in the bosonic sector. The most natural formulation of $\mathcal{N}=4, d=1$ models is achieved in the harmonic superspace $[19,20]$ parametrized by the coordinates $\left(t, \theta_{i}, \bar{\theta}^{k}, u_{i}^{ \pm}\right), i, k=1,2$, where commuting mutually conjugate SU(2)-doublets $u_{i}^{ \pm}$are harmonic coordinates, $u^{+i} u_{i}^{-}=1$. The harmonic analytic subspace is parametrized by the coordinates $(\zeta, u)=\left(t_{A}, \theta^{+}, \bar{\theta}^{+}, u_{i}^{ \pm}\right), t_{A}=$ $t-i\left(\theta^{+} \bar{\theta}^{-}+\theta^{-} \bar{\theta}^{+}\right), \theta^{ \pm}=\theta^{i} u_{i}^{ \pm}, \bar{\theta}^{ \pm}=\bar{\theta}^{i} u_{i}^{ \pm}$. The integration measures are defined as $\mu_{H}=d u d t d^{4} \theta$ and $\mu_{A}^{(-2)}=d u d \zeta^{(-2)}$.

The $\mathcal{N}=4$ supersymmetric model with $\mathrm{U}(n)$ gauge symmetry is described by the action

$$
S_{4}=S_{\chi}+S_{\mathrm{FI}}+S_{\mathrm{WZ}}
$$

The first term in (15)

$$
S_{\chi}=-\frac{1}{2} \int \mu_{H} \operatorname{Tr}\left(\chi^{2}\right)
$$

is the gauged action of the $(\mathbf{1}, \mathbf{4}, \mathbf{3})$ multiplets. The latter are described by Hermitian matrix superfields $\mathcal{X}=\left(\chi_{a}^{b}\right)$ subjected to the gauge-covariant constraints

$$
\mathcal{D}^{++} \chi=0
$$

$$
\mathcal{D}^{+} \mathcal{D}^{-} \chi=0, \quad\left(\mathcal{D}^{+} \bar{D}^{-}+\overline{\mathcal{D}}^{+} \mathcal{D}^{-}\right) \chi=0 .
$$

The constraint (17) involves the covariant harmonic derivative $\mathcal{D}^{++}=D^{++}+i V^{++}$, where the gauge matrix connection $V^{++}(\zeta, u)$ is an analytic superfield. ${ }^{1}$ The gauge connections entering the spinor covariant derivatives in (18) are properly expressed through $V^{++}(\zeta, u)$ [12]. The parameters of the $\mathrm{U}(n)$ gauge group are analytic, so $\mathcal{D}^{+}=$ $D^{+}, \overline{\mathcal{D}}^{+}=\bar{D}^{+}$. Note that $\chi$ is in the adjoint of $\mathrm{U}(n)$, so $\mathcal{D}^{++} \chi=D^{++} \chi+i\left[V^{++}, \chi\right]$, etc.

The second term in (15) is the FI term,

$$
S_{\mathrm{FI}}=\frac{i}{2} c \int \mu_{A}^{(-2)} \operatorname{Tr} V^{++} .
$$

The third term in (15),

$$
S_{\mathrm{WZ}}=\frac{1}{2} \int \mu_{A}^{(-2)} \mathcal{V}_{0} \tilde{z}^{+} Z^{+},
$$

is a WZ action describing $n$ commuting analytic superfields $Z_{a}^{+}$(analogs of the superfields $Z_{a}$ of the $\mathcal{N}=1$ and $\mathcal{N}=2$ cases). They represent off-shell $\mathcal{N}=4$ multiplets $(\mathbf{4}, \mathbf{4}, \mathbf{0})$ and are defined by the constraints

$$
\mathcal{D}^{++} Z^{+}=0, \quad D^{+} Z^{+}=0, \quad \bar{D}^{+} Z^{+}=0 .
$$

At last, the superfield $\mathcal{V}_{0}(\zeta, u)$ is a real analytic gauge superfield, $D^{+} \mathcal{V}_{0}=0, \bar{D}^{+} \mathcal{V}_{0}=0$, which is defined by the integral transform [12]

$$
\mathcal{X}_{0}\left(t, \theta_{i}, \bar{\theta}^{i}\right)=\left.\int d u \mathcal{V}_{0}\left(t_{A}, \theta^{+}, \bar{\theta}^{+}, u^{ \pm}\right)\right|_{\theta^{ \pm}=\theta^{i} u_{i}^{ \pm}, \bar{\theta}^{ \pm}=\bar{\theta}^{i} u_{i}^{ \pm}},
$$

which resolves the constraints (17) and (18) for the singlet $\mathrm{U}(1)$ part $\mathcal{X}_{0} \equiv \operatorname{Tr}(\chi)$.

The action (15) is invariant under the $\mathcal{N}=4$ superconformal group $D(2,1 ; \alpha)$ with $\alpha=-\frac{1}{2}$. To show this we should use the $D(2,1, \alpha)$ transformation laws given in $[12,20]$, in particular, that of conformal supersymmetry,

$$
\delta^{\prime} \mu_{H}=\mu_{H}\left(2 \Lambda-\frac{1+\alpha}{\alpha} \Lambda_{0}\right), \quad \delta^{\prime} \mu_{A}^{(-2)}=0,
$$

with $\Lambda=2 i \alpha\left(\bar{\eta}^{-} \theta^{+}-\eta^{-} \bar{\theta}^{+}\right), \quad \Lambda_{0}=2 \Lambda-D^{--} \Lambda^{++}$, $\Lambda^{++}=D^{++} \Lambda$. The involved $d=1$ superfields are transformed as follows:

$$
\delta^{\prime} \mathcal{X}=-\Lambda_{0} \mathcal{X}, \quad \delta^{\prime} Z^{+}=\Lambda Z^{+}, \quad \delta^{\prime} V^{++}=0 .
$$

The variation of the action (16) is vanishing only at $\alpha=$ $-\frac{1}{2}$, whereas the constraints (17), (18), and (21), as well as the actions (19) and (20), are superconformally invariant for an arbitrary parameter $\alpha$. It is important that just the field multiplier $\mathcal{V}_{0}$ in the action (20) provides this invariance due to its transformation law $\delta^{\prime} \mathcal{V}_{0}=-2 \Lambda \mathcal{V}_{0}$ [12]. Note that at $\alpha=-1 / 2$ the supergroup $D(2,1 ; \alpha)$ is isomorphic to $\mathrm{OSp}(4 \mid 2)[21,22]$, so our gauge approach in the

\footnotetext{
${ }^{1}$ Besides the covariant derivative $\mathcal{D}^{++}$which commutes with $D^{+}, \bar{D}^{+}$and so preserves the analyticity, one can define the derivative $\mathcal{D}^{--}=D^{--}+i V^{--}$, so that $\left[\mathcal{D}^{++}, \mathcal{D}^{--}\right]=D^{0}$ and $D^{0}$ is the operator counting the external U(1) charges of superfields. The nonanalytic connection $V^{--}$is expressed through $V^{++}$from this commutation relation [19].
} 
$\mathcal{N}=4$ case implies a different $\mathcal{N}=4$ superconformal group as compared to the more customary $\mathrm{SU}(1,1 \mid 2)$ used e.g. in [9].

The local $\mathrm{U}(n)$ transformations leaving the action (15) invariant are given by

$$
\begin{gathered}
\chi^{\prime}=e^{i \lambda} \chi e^{-i \lambda}, \quad Z^{+\prime}=e^{i \lambda} Z^{+}, \\
V^{++\prime}=e^{i \lambda} V^{++} e^{-i \lambda}-i e^{i \lambda}\left(D^{++} e^{-i \lambda}\right),
\end{gathered}
$$

where $\lambda_{a}^{b}\left(\zeta, u^{ \pm}\right) \in u(n)$ is the "Hermitian" analytic matrix parameter, $\tilde{\lambda}=\lambda$. Using this gauge freedom we can choose the WZ gauge

$$
V^{++}=-2 i \theta^{+} \bar{\theta}^{+} A\left(t_{A}\right) .
$$

In this gauge we have

$$
\begin{gathered}
\mathcal{D}^{ \pm \pm}=D^{ \pm \pm}+2 \theta^{ \pm} \bar{\theta}^{ \pm} A, \quad \mathcal{D}^{-}=D^{-}-2 \bar{\theta}^{-} A, \\
\overline{\mathcal{D}}^{-}=\bar{D}^{-}-2 \theta^{-} A,
\end{gathered}
$$

and the constraints (17) and (18) are solved by

$$
\chi=X+\theta^{-} \bar{\theta}^{-} N^{++}+\theta^{-} \Psi^{+}+\bar{\theta}^{-} \bar{\Psi}^{+}+\ldots,
$$

where $N^{++}=N^{i k} u_{i}^{+} u_{k}^{+}, \Psi^{+}=\Psi^{i} u_{i}^{+}, \bar{\Psi}^{+}=\bar{\Psi}^{i} u_{i}^{+}$and the fields $X\left(t_{A}\right), N^{i k}=N^{(i k)}\left(t_{A}\right), \Psi^{i}\left(t_{A}\right), \bar{\Psi}^{i}\left(t_{A}\right)$ are ordinary $d=1$ fields having no dependence of the harmonics. All other fields in (25) are expressed through these fields and their covariant derivatives $\nabla_{t_{A}} X=\partial_{t_{A}} X+i[A, X]$, etc. The solution of the constraints (21) is

$$
Z^{+}=Z^{+}+\theta^{+} \varphi+\bar{\theta}^{+} \phi+2 i \theta^{+} \bar{\theta}^{+} Z^{-},
$$

where $Z^{+}=Z^{i}\left(t_{A}\right) u_{i}^{+}, Z^{-}=\nabla_{t_{A}} Z^{i}\left(t_{A}\right) u_{i}^{-}$.

Inserting the expressions (25) and (26) in the action (15) and eliminating the fields $N^{i k}, \phi, \bar{\phi}, \varphi, \bar{\varphi}$ by their equations of motion we obtain, in the WZ gauge,

$$
\begin{gathered}
S_{4}=S_{b}+S_{f}, \\
S_{b}=\int d t\left[\operatorname{Tr}(\nabla X \nabla X+c A)+\frac{n}{8}\left(\bar{Z}^{(i} Z^{k)}\right)\left(\bar{Z}_{i} Z_{k}\right)\right. \\
\left.+\frac{i}{2} X_{0}\left(\bar{Z}_{k} \nabla Z^{k}-\nabla \bar{Z}_{k} Z^{k}\right)\right] \\
S_{f}=-i \operatorname{Tr} \int d t\left(\bar{\Psi}_{k} \nabla \Psi^{k}-\nabla \bar{\Psi}_{k} \Psi^{k}\right) \\
-\int d t \frac{\Psi_{0}^{(i} \bar{\Psi}_{0}^{k)}\left(\bar{Z}_{i} Z_{k}\right)}{X_{0}}
\end{gathered}
$$

where

$$
X_{0} \equiv \operatorname{Tr}(X), \quad \Psi_{0}^{i} \equiv \operatorname{Tr}\left(\Psi^{i}\right), \quad \bar{\Psi}_{0}^{i} \equiv \operatorname{Tr}\left(\bar{\Psi}^{i}\right) .
$$

Let us consider the bosonic limit of $S_{4}$, i.e. the action (28). We can impose the gauge $X_{a}^{b}=0, a \neq b$, using the residual invariance of the WZ gauge (23): $X^{\prime}=e^{i \lambda} X e^{-i \lambda}$, $Z^{\prime k}=e^{i \lambda} Z^{k}, A^{\prime}=e^{i \lambda} A e^{-i \lambda}-i e^{i \lambda}\left(\partial_{t} e^{-i \lambda}\right)$, where $\lambda_{a}^{b}(t) \in$ $u(n)$ are ordinary $d=1$ gauge parameters. As a result of this, and after eliminating $A_{a}^{b}, a \neq b$, by the equations of motion, the action (28) takes the following form [instead of $Z_{a}^{i}$ we introduce the new fields $Z_{a}^{i i}=\left(X_{0}\right)^{1 / 2} Z_{a}^{i}$ and omit the primes on these fields],

$$
\begin{aligned}
S_{b}= & \int d t\left\{\sum_{a} \dot{x}_{a} \dot{x}_{a}+\frac{i}{2} \sum_{a}\left(\bar{Z}_{k}^{a} \dot{Z}_{a}^{k}-\dot{\bar{Z}}_{k}^{a} Z_{a}^{k}\right)\right. \\
& \left.+\sum_{a \neq b} \frac{\operatorname{Tr}\left(S_{a} S_{b}\right)}{4\left(x_{a}-x_{b}\right)^{2}}-\frac{n \operatorname{Tr}(\hat{S} \hat{S})}{2\left(X_{0}\right)^{2}}\right\} .
\end{aligned}
$$

Here, the fields $Z_{a}^{k}$ are subject to the constraints ${ }^{2}$

$$
\bar{Z}_{i}^{a} Z_{a}^{i}=c \quad \forall a,
$$

and carry the residual Abelian gauge $[\mathrm{U}(1)]^{n}$ symmetry, $Z_{a}^{k} \rightarrow e^{i \varphi_{a}} Z_{a}^{k}$, with local parameters $\varphi_{a}(t)$. In (30) we use the following notation:

$$
\left(S_{a}\right)_{i}{ }^{j} \equiv \bar{Z}_{i}^{a} Z_{a}^{j},
$$

$$
(\hat{S})_{i}{ }^{j} \equiv \sum_{a}\left[\left(S_{a}\right)_{i}{ }^{j}-\frac{1}{2} \delta_{i}^{j}\left(S_{a}\right)_{k}{ }^{k}\right] .
$$

Note that at $c=0$ the constraint (31) implies $Z_{a}^{i}=0$, i.e. a nontrivial interaction exists only for $c \neq 0$ as in the previous cases. The new feature of the $\mathcal{N}=4$ case is that not all of the bosonic variables $Z_{a}^{i}$ are eliminated by fixing gauges and solving the constraint; there survives a nonvanishing WZ term for them in Eq. (30). After quantization these variables become purely internal [U(2)-spin] degrees of freedom.

In the Hamiltonian approach, the kinetic WZ term for $Z$ in (30) gives rise to the following Dirac brackets:

$$
\left[\bar{Z}_{i}^{a}, Z_{b}^{j}\right]_{D}=i \delta_{b}^{a} \delta_{i}^{j} .
$$

With respect to these brackets the quantities (32) for each index $a$ form $u(2)$ algebras

$$
\left[\left(S_{a}\right)_{i}{ }^{j},\left(S_{b}\right)_{k}{ }_{k}\right]_{D}=i \delta_{a b}\left\{\delta_{i}^{l}\left(S_{a}\right)_{k}{ }^{j}-\delta_{k}^{j}\left(S_{a}\right)_{i}{ }^{l}\right\} .
$$

The quantities (33) are time-independent Noether charges for the $\mathrm{SU}(2)$ invariance of the system (30), so the numerator of the term $\sim\left(X_{0}\right)^{-2}$ in (30) is a constant on the equations of motion for $Z_{a}^{i}, \bar{Z}_{i}^{a}$. So, as opposed to the $\mathcal{N}=$ 1,2 , cases, the $\mathcal{N}=4$ action contains a conformal potential even in the center-of-mass sector (like in $[9,11]$ ). Up to this extra conformal potential [last term in (30)], the bosonic limit of the $\mathcal{N}=4$ system constructed is none other than the integrable U(2)-spin Calogero model [17] in the formulation of $[3,18]$.

While the coordinate $X_{0}$ decouples in the bosonic limit, when all fermions are discarded, this is not the case for the full action because of the term $\sim X_{0}^{-1}$ in (29). The full $\mathrm{SU}(2)$ current contains extra fermionic terms, and its bosonic part (33) is not conserved by itself.

\footnotetext{
${ }^{2}$ Here and in (32) we do not sum over the repeated index $a$.
} 


\section{OUTLOOK}

In this paper we proposed a new gauge approach to the construction of superconformal Calogero-type systems as a superextension of the bosonic construction of [13]. The characteristic features of this approach are the presence of auxiliary supermultiplets with WZ-type actions, the builtin superconformal invariance, and the emergence of the Calogero coupling constant as a strength of the FI term of the $\mathrm{U}(1)$ gauge (super)field. Here we used the $\mathrm{U}(n)$ gauging and obtained new superextensions of the $A_{n-1}$ Calogero model and of its $\mathrm{U}(2)$-spin extension (in the $\mathcal{N}=4$ case). Superextensions of other conformal Calogero models could be obtained presumably by choosing other gauge groups and/or representations for the matrix and WZ superfields. Superextensions of nonconformal models can be constructed by adding proper gauge invariant (but not conformally invariant) potentials to the original superfield actions.

While in the $\mathcal{N}=1$ and $\mathcal{N}=2$ cases there is almost no freedom in the choice of the original gauged action (provided that it is required to be minimal and superconformal), it is not so in the $\mathcal{N}=4$ case due to the diversity of the $\mathcal{N}=4, d=1$ multiplets. For instance, any sort of the $\mathcal{N}=4, d=1$ multiplet has its "mirror" in which another $\mathrm{SU}(2)$ from the full $R$-symmetry group $\mathrm{SO}(4)$ of the $\mathcal{N}=4, d=1$ superalgebra is manifest. We are planning to consider these possibilities elsewhere.

In the $\mathcal{N}=4$ case we used as the basic matrix superfield the new non-Abelian version of the multiplet $(\mathbf{1}, \mathbf{4}, \mathbf{3})$ defined by the constraints (17) and (18). Its simplest, quadratic action is invariant under the superconformal group $D(2,1 ; \alpha)$ with $\alpha=-1 / 2$ [which is isomorphic to $\mathrm{OSp}(4 \mid 2)]$. It is worth noting that our gauging procedure is compatible as well with other $\mathcal{N}=4$ superconformal groups [20-23]. For any value of $\alpha \neq 0$ the superconformal $\mathcal{N}=4$ gauged action has the generic form of (15) with the same $S_{\mathrm{WZ}}$ and $S_{\mathrm{FI}}$, the only difference being in the form of the action for $\mathcal{X}$,

$$
S_{\chi}^{\alpha \neq 0}=\alpha \int \mu_{H}\left[\operatorname{Tr}\left(\chi^{2}\right)\right]^{-1 /(2 \alpha)} .
$$

It is important that $\mathcal{X}$ is a matrix and, therefore, this action is nontrivial even in the case of $\alpha=-1$ as opposed to the standard case of the Abelian $(\mathbf{1}, \mathbf{4}, \mathbf{3})$ multiplet. The second possibility at $\alpha=-1$ is to consider the matrix version of the standard conformal action

$$
\tilde{S}_{\chi}^{\alpha=-1}=\int \mu_{H} \operatorname{Tr}(\chi \ln \chi)
$$

It seems, however, that all such actions except for the case of $\alpha=-1 / 2$ yield nontrivial sigma-model-type kinetic terms for the $X$ fields, so the corresponding bosonic limits are more general conformal models.

\section{ACKNOWLEDGMENTS}

We thank Francois Delduc and Armen Nersessian for the interest in this work. We acknowledge support from a grant of the Heisenberg-Landau Programme, RFBR Grants No. 06-02-16684 and No. 08-02-90490, INTAS Grant No. 05-1000008-7928 (S.F. and E.I.), and a DFG grant, Project No. 436 RUS/113/669 (E. I. and O. L.).
[1] F. Calogero, J. Math. Phys. (N.Y.) 10, 2191 (1969); 10, 2197 (1969); 12, 419 (1971).

[2] M. A. Olshanetsky and A. M. Perelomov, Phys. Rep. 71, 313 (1981); 94, 313 (1983).

[3] A. P. Polychronakos, J. Phys. A 39, 12793 (2006).

[4] G. W. Gibbons and P. K. Townsend, Phys. Lett. B 454, 187 (1999).

[5] D. Z. Freedman and P. F. Mende, Nucl. Phys. B344, 317 (1990).

[6] N. Wyllard, J. Math. Phys. (N.Y.) 41, 2826 (2000).

[7] S. Bellucci, A. Galajinsky, and S. Krivonos, Phys. Rev. D 68, 064010 (2003).

[8] S. Bellucci, A. V. Galajinsky, and E. Latini, Phys. Rev. D 71, 044023 (2005).

[9] A. Galajinsky, O. Lechtenfeld, and K. Polovnikov, Phys. Lett. B 643, 221 (2006); J. High Energy Phys. 11 (2007) 008; 03 (2009) 113.

[10] S. Bellucci, S. Krivonos, and A. Sutulin, Nucl. Phys. B805, 24 (2008).
[11] S. Krivonos, O. Lechtenfeld, and K. Polovnikov, arXiv:0812.5062.

[12] F. Delduc and E. Ivanov, Nucl. Phys. B753, 211 (2006); B770, 179 (2007); B787, 176 (2007); Phys. Lett. B 654, 200 (2007)

[13] A. P. Polychronakos, Phys. Lett. B 266, 29 (1991).

[14] A. Gorsky and N. Nekrasov, Nucl. Phys. B414, 213 (1994); B436, 582 (1995); Theor. Math. Phys. 100, 874 (1994).

[15] A. P. Polychronakos, J. High Energy Phys. 04 (2001) 011.

[16] B. Morariu and A. P. Polychronakos, J. High Energy Phys. 07 (2001) 006; Phys. Rev. D 72, 125002 (2005).

[17] J. Gibbons and T. Hermsen, Physica (Amsterdam) 11D, 337 (1984); S. Wojciechowski, Phys. Lett. 111A, 101 (1985).

[18] A. P. Polychronakos, Nucl. Phys. B543, 485 (1999); Phys. Rev. Lett. 89, 126403 (2002).

[19] A. S. Galperin, E. A. Ivanov, V. I. Ogievetsky, and E. S. Sokatchev, Harmonic Superspace (Cambridge University 
Press, Cambridge, England, 2001), p. 306.

[20] E. Ivanov and O. Lechtenfeld, J. High Energy Phys. 09 (2003) 073.

[21] L. Frappat, A. Sciarrino, and P. Sorba, arXiv:hep-th/
9607161.

[22] A. Van Proeyen, arXiv:hep-th/9910030.

[23] E. Ivanov, S. Krivonos, and O. Lechtenfeld, J. High Energy Phys. 03 (2003) 014. 